\title{
Efficient Design of Rectifying Antennas for Low Power Detection
}

\author{
Vlad Marian ${ }^{1}$, Cyrille Menudier ${ }^{2}$, Marc Thevenot ${ }^{2}$, Christian Vollaire ${ }^{1}$, Jacques Verdier ${ }^{1}$, Bruno Allard ${ }^{1}$ \\ ${ }^{1}$ Université de Lyon, Laboratoire Ampère, CNRS UMR 5005, Ecully, France \\ ${ }^{2}$ Université de Limoges, XLIM - OSA, CNRS UMR 6172, Limoges, France
}

\begin{abstract}
This article is dedicated to the design of rectifying antenna for wireless energy transfer at $2.45 \mathrm{GHz}$ in the special case of low input power (typically $<-10 \mathrm{dBm}$ ). As the rectifiers present an important non-linear behavior with such power levels, specific design guidelines must be respected in order to optimize the structure. Different rectifying circuits have been studied and their performances have been evaluated through the definition of a Figure of Merit. The effects of the antenna impedance have also been investigated to improve the performances. Two test-circuits have been manufactured and measured to validate the simulation results and to confirm the interest of a global design approach with such rectifying structures.
\end{abstract}

Index Terms - Rectenna, RF to DC conversion, Wireless Energy Transfer, Nonlinear circuit.

\section{INTRODUCTION}

Defined as the process of sending electrical energy from one point to another without the use of a conducting environment, wireless energy transfer can be realized using mainly two different approaches. The first consists in using the magnetic resonant coupling between two structures, usually circular coils [1]. The energy transfer is done in the near field area and frequencies used are relatively low. The main inconvenient of this method is that energy can only be transferred over relatively low distances, generally in the same order of magnitude as emitter and receiver size.

The second possibility is based on a radiative high frequency energy transfer. It uses high frequency electromagnetic waves, often above $1 \mathrm{GHz}$, and energy transfer is done in the far field region. This method allows both short and long range wireless energy transfer with relatively compact emitter and receiver sizes. The process is done in three main stages. First, the high frequency power signal is generated and sent by the emitter antenna. Power is transported under the form of an electromagnetic wave beam towards the receiver, where it is captured by the receiver antenna and transformed into DC power by rectifying structures. The association of a receiving antenna and an RF to DC rectifier circuit is called a rectenna ("rectifying antenna") and it is a critical component of the energy transfer system.

The transfer of large quantities of energy wirelessly over large distances is a concept that was first introduced by Tesla, as early as the beginning of the $20^{\text {th }}$ century, but practical demonstrations began to appear in the 1950s and the 1960s, in military and space-related research programs like the Solar Power Satellite (SPS) project or the Raytheon Airborn Microwave Platform (RAMP) project [2], [3]. The objective of these projects is to transfer energy amounts ranging from several watts to several kilowatts over distances reaching several kilometers in some cases. RF to DC conversion efficiencies in excess of $70 \%$ are sometimes reported at these power levels [4], [5], but the number of viable applications tends to be limited due to strict electromagnetic health and safety regulations and large antenna sizes.

The concept of wireless energy transfer can also be applied in order to supply low power electronic devices like sensors or RFID tags over distances of several meters. These devices can either be supplied exclusively by the energy from the microwave beam [6] or by batteries that can be remotely recharged [7]. In certain cases, high sensitivity rectennas have been reported to harvest power amounts in the $\mu \mathrm{W}$ range from ambient electromagnetic radiation generated by cellular phone base stations or WLAN access points [8], [9].

In this paper, several designs of rectennas for low input power (below $-10 \mathrm{dBm}$, typically $-15 \mathrm{dBm}$ ) are investigated for wireless energy transfer. The rectifying antennas studied are dedicated to small terminal without any power supply allowing the use of a complementary low level detector. That is why the design of the rectifying structure must be fully optimized to obtain satisfactory link budget. As a whole, each element has to be precisely considered in simulation (nonlinear diode model, lumped components accuracy and package effects, complex impedances). All of them may have considerable repercussions on overall rectenna performances.

\section{TECHNOLOGY AND CIRCUIT CONFIGURATION}

Antenna aside, RF to DC conversion circuits are built around diodes or diode-mounted transistors. Diode threshold voltage has to be overcome in order to put them in a conductive state; it is a key factor when choosing a diode for designing a rectenna. Under high power levels, diode threshold is not an issue because it is small compared to incident high frequency voltage amplitude, but losses due to diode threshold become predominant in the case of low incident power levels (below $1 \mathrm{~mW}$ ) [10]. We chose to use zero bias Schottky diodes due to their low threshold of 150 $\mathrm{mV}$ and their low junction capacitance of around $0.18 \mathrm{pF}$.

In a typical rectenna circuit (Fig. 1) incident RF power captured by the antenna is transformed into DC power by the diode based converter. A HF filter ensures impedance match between the rectifier and the antenna for optimal power transfer. The output DC filter smoothens the output DC voltage and current by attenuating high frequency harmonics present in the RF signal or generated by the highly nonlinear rectification process. 


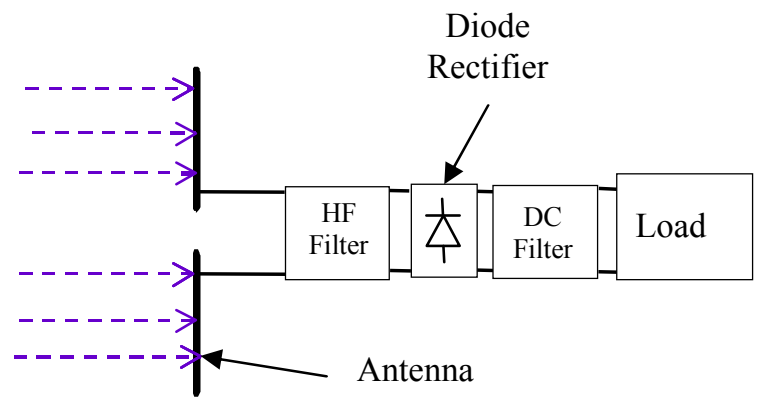

Fig. 1. Block diagram of rectenna circuit

When designing a rectenna, two main parameters are generally evaluated: DC voltage output and RF to DC energy conversion efficiency, defined as:

$$
\eta=\frac{P_{D C \text { out }}}{P_{R F \text { in }}}=\frac{V_{\text {out }}^{2}}{R_{\text {load }}} \cdot \frac{4 \pi \cdot Z_{\text {air }}}{|E|^{2} \cdot G \cdot \lambda^{2}}
$$

where $R_{\text {load }}$ is the load resistance, $Z_{\text {air }}$ is air characteristic impedance $(120 \pi$ ohms), E is electric field RMS value at receiver position, $\mathrm{G}$ is receiver antenna gain and $\lambda$ is the wavelength.

Rectenna circuits can have different topologies, depending on the configuration of the diodes used for the rectification. Standard bridge topology that is widely used for low frequency rectification has proven to be unsuitable for low power rectenna applications [11]. Single series and shunt mounted diode structures theoretically offer the advantage of minimizing diode loss, which is proportional to diode junction resistance. Other structures like voltage doublers can generally reach higher DC voltage levels for the same input power, in the detriment of power conversion efficiency. Designers are often confronted with the choice of a compromise between high output voltage and good conversion efficiency.

For the purpose of providing an overall comparison tool that includes both the above mentioned parameters, a Rectenna Figure of Merit (RFoM) can be defined as:

$$
R F o M\left(P_{\text {in }}\right)=V_{D C \text { open } \text { circuit }} \cdot \eta_{\text {optimal load }}
$$

Simulations have allowed us to assess the Figure of Merit of four different structures: series and shunt mounted single diode configurations as well as a single stage and a two stage voltage doubler circuit topology, as summarized on Fig. 2. Simulated data at an input frequency of $2.45 \mathrm{GHz}$ is presented in Fig. 3.

Voltage doubler topologies have low RFoM due mainly to their low energy conversion efficiency for low power levels. However, the two stage voltage doubler has high RFoM for high input power levels due to its high DC output voltage. The single diode topologies are similar for power levels of around $30 \mu \mathrm{W}$, but the series mounted diode rectifier seems to have the highest RFoM for low input power levels (below $-5 \mathrm{dBm}$ ). It thus offers the best compromise between DC output level and conversion efficiency.

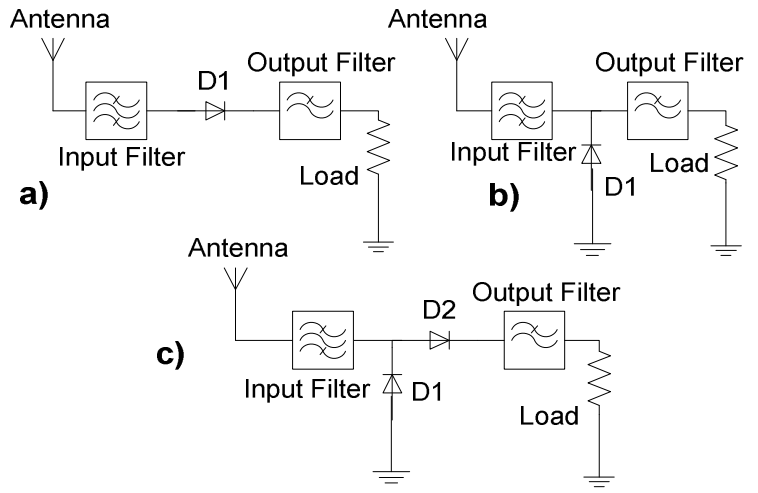

Fig. 2. Rectenna circuit topologies: a) series; b) shunt; c) single stage voltage doubler

Voltage doubler topologies have low RFoM due mainly to their low energy conversion efficiency for low power levels. However, the two stage voltage doubler has high RFoM for high input power levels due to its high DC output voltage. The single diode topologies are similar for power levels of around $30 \mu \mathrm{W}$, but the series mounted diode rectifier seems to have the highest RFoM for low input power levels (below $-5 \mathrm{dBm}$ ). It thus offers the best compromise between DC output level and conversion efficiency.

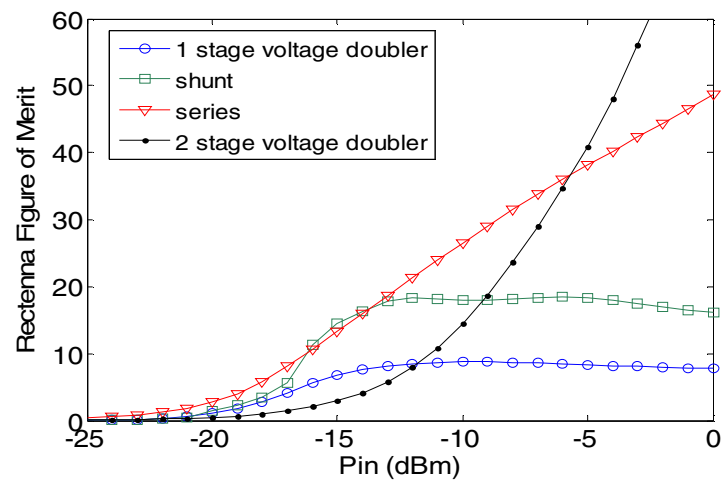

Fig. 3. Simulated Rectenna Figure of Merit (in Volts) as a function of input power level

The decision was made to focus the next parts of the article on this single series diode structure.

\section{RECTENNA DESIGN}

Rectenna circuits have a highly non linear behavior due to the presence of diodes. For this reason, it is impractical to design its subparts like input and output filters independently from each other. In fact, the input impedance of the diode loaded by an output filter and a specific load is affected if different input passive circuits (filters) are used before the diode. This effect is presented on Fig. 4 where circuit is best adapted at $-15 \mathrm{dBm}$ input power when a global optimization at this power level is done, compared to $-19 \mathrm{dBm}$ when filter is 
designed using the filter design tool, which supposes the circuit as linear.

As a whole a global circuit optimization techniques must be used for dimensioning passive component values of the filter elements. Moreover, the effect of the printed lines has to be taken into account from a RF point-of-view because they also affect the circuit matching. These optimizations have been performed with the software ADS (and Momentum) from Agilent Technologies.

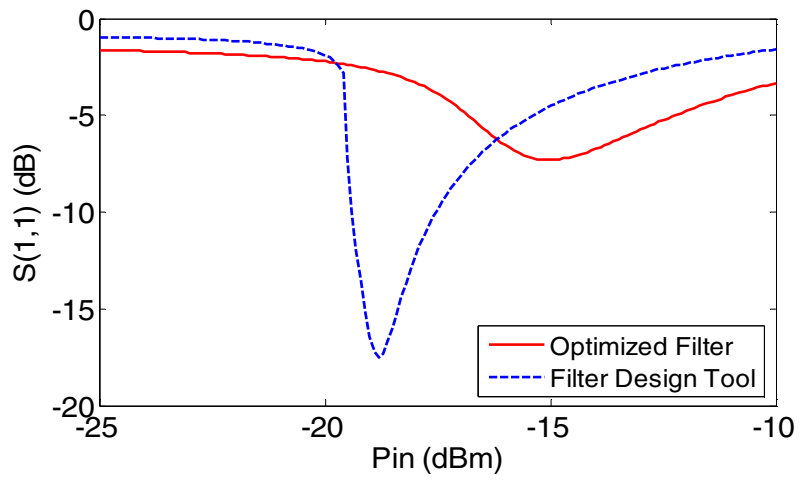

Fig. 4. Comparison of return loss between rectenna circuit with optimized filter and filter designed with the filter design tool

Traditionally, the impedance matching between receiver antenna and rectification circuit is done using the $50 \mathrm{Ohm}$ standard in order to simplify testing. With such a classical design (50 Ohm input impedance for the antenna) and with a global optimization of the rectifying circuit, we obtain a simulated voltage level of $350 \mathrm{mV}$ on a 1 MOhm load (representative of a high impedance connected sub-circuit) and for an input power level of $-15 \mathrm{dBm}$. RF do DC conversion efficiency reaches $25 \%$ when supplying a $3 \mathrm{kOhm}$ resistive load.

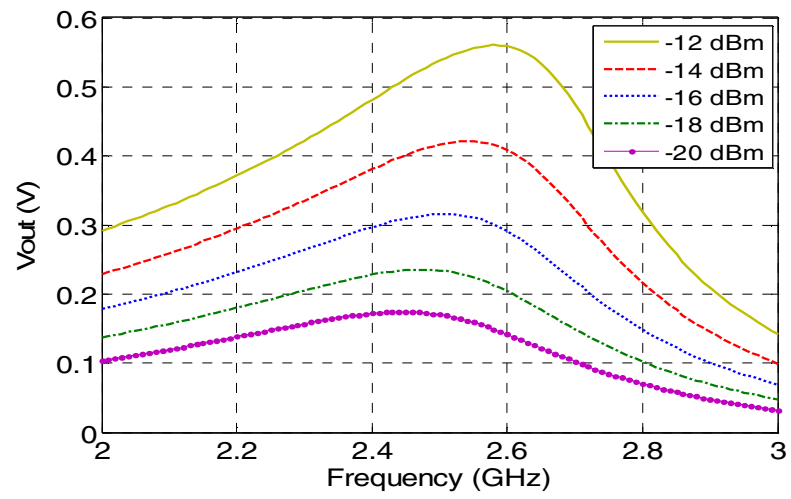

Fig. 5. Output DC voltage of the rectenna circuit for different input power levels

Fig. 5 illustrates the highly nonlinear behavior of the rectenna circuit. Output DC voltage is represented as a function of incident power frequency, for different input power levels. Other than the fact that maximum voltage level increases with input power, the central frequency at which this maximum is obtained is also power-level dependent. Maximum DC voltage is reached at $2.43 \mathrm{GHz}$ for $-20 \mathrm{dBm}$ input power, while this maximum shifts to $2.6 \mathrm{GHz}$ at -12 $\mathrm{dBm}$.

This is an illustration of the fact that all design parameters are inter-dependent and that the only efficient approach is a global optimization of the whole structure for each specific application scenario.

Once all the structure is optimized for optimal RF to DC conversion efficiency and high output voltage level, we evaluated the impact of a non $50 \mathrm{Ohm}$ antenna on the overall circuit performances. Fig. 6 traces the evolution of output DC voltage level as a function of antenna internal impedance.

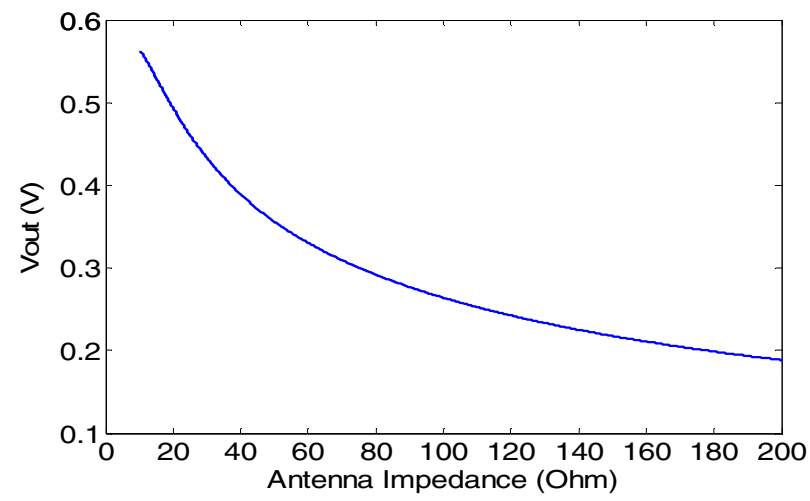

Fig. 6. Simulated influence of the antenna impedance on rectenna DC output voltage

It appears that for this particular structure, lower antenna impedance is more adequate and allows an increase of output voltage level from around $350 \mathrm{mV}$ with a $50 \mathrm{Ohm}$ antenna to more than $500 \mathrm{mV}$ with $10 \mathrm{Ohm}$ antenna impedance. Antenna characteristic impedance is thus added as a degree of freedom in circuit optimization. This result is very important by considering the fact that with the low input power level, every additional degree of freedom can significantly improve the performances, and thus the link budget. After a new optimization, output voltage in the vicinity of $550 \mathrm{mV}$ and conversion efficiency in excess of $35 \%$ was obtained with this structure.

\section{MEASUREMENT RESULTS}

In order to validate the aforementioned results, two different circuits have been designed and manufactured. They are both based on the single series diode structure. To avoid a difficult realization process of the antenna which may lead to inaccurate results, we have chosen to represent the antenna behavior by a RF generator connected to a simple L-C matching cell connected to the rectifier. By this way, the effect of the antenna impedance modification is experimentally simulated and allows a direct comparison with simulations.

The first circuit is a reference case because it corresponds to a $50 \mathrm{Ohm}$ input impedance design, i.e. with non-optimized antenna impedance. The circuit has been simulated with the 
guidelines presented before, especially concerning the necessity to perform a global simulation.

The second circuit has been optimized by including the antenna impedance as a degree of freedom. The two circuits are presented on Fig. 7 and the corresponding manufactured prototypes in Fig. 8. Performances are shown on Fig. 9.

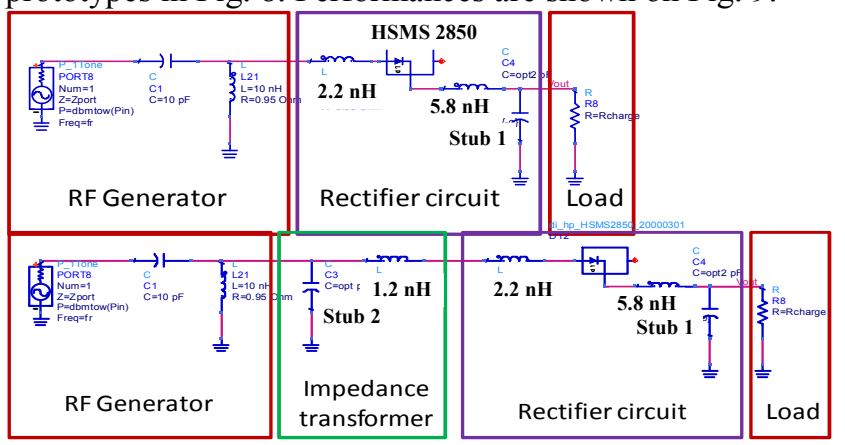

Fig. 7. Reference circuit with $50 \mathrm{Ohm}$ input impedance (top); and fully optimized rectifier with non-50 Ohm impedance (bottom)

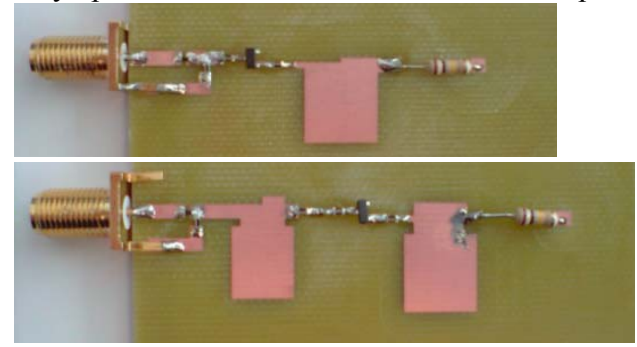

Fig. 8. Pictures of the realized reference circuit with $50 \mathrm{Ohm}$ input impedance (top) and fully optimized rectifier (bottom)

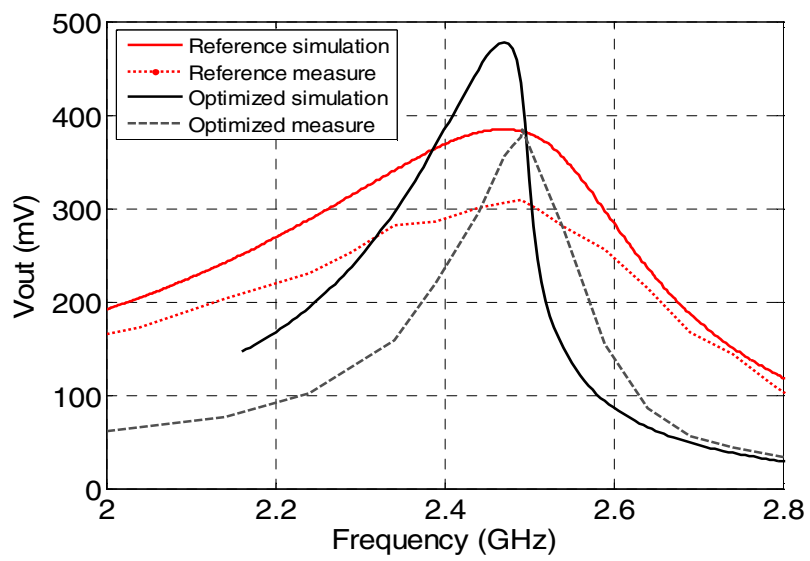

Fig. 9. Improvement of performances due to the global optimized design compared to the reference one (classical design)

A $1.6 \mathrm{~mm}$ thickness FR-4 substrate has been used for the prototypes $\left(\varepsilon_{\mathrm{r}}=4.3, \tan \delta=0.02\right)$. The lumped elements come from AVX for the capacitors and Tyco Electronics for the inductors. Notice that these components have been selected because they were frequency compliant at $2.45 \mathrm{GHz}$. The manufactured circuits also used printed circuit capacitors. This choice has been retained to minimize the accuracy dispersion of an equivalent lumped component.

The results on Fig. 9 validate the different guidelines applied to the design. Although maximum DC voltage level is different between simulation and measurement (around 100 $\mathrm{mV}$ ), a relative comparison between the reference circuit and the optimized one leads to a great compliance between the predicted result and the measurement. As a whole, an improvement of output voltage of around $100 \mathrm{mV}$ is observed when the antenna impedance is added to the design optimization. Notice that this last design leads to a more selective behavior of the rectenna.

\section{CONCLUSION}

Different rectifying antenna structures have been studied and compared for wireless energy transfer with very low input power $(<-10 \mathrm{dBm})$. The effects of non linearity and antenna impedances have lead to guidelines for efficient design. A comparison with measurement has proven the interest of the method used to conceive the rectifier. Other works are currently performed to offer a greater performance improvement compared to classical design.

\section{REFERENCES}

[1] A. Karalis, J. D. Joannopoulos, and M. Soljacic, "Efficient Wireless Non-Radiated Mid-Range Energy Transfert," Annals of Physics, vol. 323, pp. 34-48, 2008.

[2] C. Cougnet, E. Sein, A. Celeste and L. Summerer, "Solar Power Satellites for Space Applications," 55 $5^{\text {th }}$ International Astronautical Congress, Vancouver, 2004.

[3] W. C. Brown, "The History of Wireless Power Transmission," Solar Power, vol. 56, pp. 3-21, 1996.

[4] W. C. Brown, "Optimization of the Effeciency and Other Properties of the Rectenna Element", MTT-S International Microwave Symposium Digest of Technical Papers, Vol. 76, N¹, pp. 142-144, 1976.

[5] Y. J. Ren and K. Chang, "5.8-GHz Circularly Polarized Dual-Diode Rectenna and Rectenna Array for Microwave Power Transmission", IEEE Trans. On Microwave Theory and Techniques, Vol. 54, N 4, pp. 1495-1502, April 2006.

[6] A. Ashry, K. Sharaf and M. Ibrahim, "A compact low-power UHF RFID tag," Microelectronics Journal, vol. 40, pp. 1504-1513, 2009.

[7] J. Essel, D. Brenk, J. Heidrich, H. Reinisch, G. Hofer, G. Holweg and R. Weigel, "Highly efficient multistandard RFIDs enabling passive wireless sensing," Microwave Conference Asia Pacific, pp. 2228 - 2231, 7-10, 2009.

[8] J. Hagerty, F. Helmbrecht, W. McCalpin, R. Zane and Z. Popovic, "Recycling Ambient Microwave Energy with Broad-Band Rectenna Arrays," IEEE Transactions on Microwave Theory and Techniques, Vol. 52, No. 3, 2004

[9] R. Vullers, H. Visser, B. Op het Veld and V. Pop, "RF Harvesting Using Antenna Structures on Foil," Proceedings of PowerMEMS 2008+microEMS2008, Sendai-Japan, November 9-12, 2008.

[10] B. Merabet, H. Takhedmit, B. Allard, L. Cirio, F. Costa, O. Picon, C. Vollaire, "Low-cost converter for harvesting of microwave electromagnetic energy", IEEE Energy Conversion Congress and Exposition, San Jose, 2009.

[11] B. Merabet, F. Costa, H. Takhedmit, C. Vollaire, B. Allard, L. Cirio, O. Picon, "A $2.45 \mathrm{GHz}$ localized elements rectenna," 3rd IEEE International Symposium on Microwave, Antenna, Propagation and EMC Technologies for Wireless Communications, 2009. 